\title{
Application of Left Axillary Incision in Patent Ductus Arteriosus Ligation
}

\author{
Aijun Liu, Ming Yang, Xiangming Fan, Bin Li, Junwu Su \\ Pediatric Cardiac Center, Beijing Anzhen Hospital, Capital Medical University, Beijing, China
}

\section{ABSTRACT}

Background: Left posterolateral incision has been a conventional incision for patent ductus arteriosus ligation. This study aimed to evaluate the efficacy and safety of left axillary thoracotomy for patent ductus arteriosus ligation.

Methods: Between January 2013 and December 2019, the clinical data of 76 patients who underwent left axillary thoracotomy for patent ductus arteriosus ligation were compared with the data of a paired group of 101 patients who underwent left posterolateral thoracotomy.

Results: Compared with the left posterolateral group, the left axillary group showed less drainage $(\mathrm{P}<0.05)$. Operation time, postoperative mechanical ventilation time, and postoperative hospitalization duration were similar between the groups. Complications were rare in both groups with no mortality during follow up. In total, 72 patient families (95\%) in the left axillary group and 81 patient families $(80 \%)$ in the left posterolateral group were satisfied with their cosmetic results $(\mathrm{P}<0.01)$.

Conclusions: A left axillary thoracotomy is as safe and effective as a left posterolateral thoracotomy for patent ductus arteriosus ligation. With lower trauma and better cosmetic results, this procedure provides a good alternative to the standard left posterolateral thoracotomy.

\section{INTRODUCTION}

Patent ductus arteriosus is one of the most common congenital heart diseases, accounting for about $5-10 \%$ of all congenital heart diseases [Dice 2007; Schneider 2012]. Closure of the arterial ductus is recommended to be completed before the age of 2 to reduce the occurrence of infective endocarditis and pulmonary hypertension [Heuchan 2014; Mitra 2013]. For patent ductus arteriosus that is ineffective in medical treatment, ductus arteriosus ligation is the conventional therapy [Garcia 2017]. Minimally invasive techniques, such as interventional therapy, thoracoscopy and robotic surgery, are more and more used in the therapy of patent ductus arteriosus to obtain better aesthetic results [Baruteau 2014; Jin 2015;

Received October 24, 2020; received in revised form November 24, 2020; accepted November 24, 2020

Correspondence: Junwu Su, Pediatric Cardiac Center, Beijing Anzhen Hospital, Capital Medical University, No. 2 Anzhen Rd, Chaoyang District, Beijing 100029, China, Telephone +86-10-64456093, Fax +86-10-64456093 (e-mail: sujunwu@ccmu.edu.cn)
Nezafati 2011; Suematsu 2005]. They are proven to be safe and effective, but they have limited indications and higher costs. Surgical ligation often uses a left posterolateral incision to reduce the trauma of the median incision and avoid median scars [Susheel 2019].

Our center uses the left axillary incision instead of the traditional left posterolateral incision. This article compares the clinical and aesthetic effects of the left axillary incision and the traditional left posterolateral incision.

\section{METHODS}

Patient selection: From January 2013 to December 2019, 187 patients in Beijing Anzhen Hospital underwent simple patent ductus arteriosus ligation. Among them, 10 cases were operated through median incision, 76 cases were operated through left axillary incision (left axillary group), and 101 cases were operated through left posterolateral incision (left posterolateral group). The choice of incision was decided by the doctor and the patient family. For patients with mild or moderate pulmonary hypertension, a left axillary or left posterolateral incision was used. Patients with severe pulmonary hypertension were treated through a median incision. The baseline data of patients with left axillary incision and left posterolateral incision were collected before surgery; data included age, gender, weight, diagnosis, diameter of arterial ductus arteriosus, etc.

This retrospective study was approved by the Medical Ethics Committee of Beijing Anzhen Hospital and the need for patient consent to publish the perioperative data from these patients was waived.

Operative technique: In the operation, venous inhalation anesthesia at room temperature was used, and the blood pressure was continuously monitored by placing a catheter in the artery of the lower extremity. The surgical technique of the left axillary incision is similar to the previously published right axillary incision to treat simple congenital heart diseases. The patient is placed in the right decubitus position, and the left side is elevated $60^{\circ}$ to $80^{\circ}$. The left arm is raised and fixed above the side of the head. The anterior axillary line (as the front boundary of the incision) and the posterior axillary line are marked. The length of the incision is 5 to $10 \mathrm{~cm}$, the upper edge is in the third intercostal space, and the lower edge is in the sixth intercostal space. Cut open the skin, subcutaneous tissue, and anterior fascia of the latissimus dorsi, enter the thoracic cavity through the third or fourth intercostal space between the serratus anterior muscle branches, and use two retractors to help expose the visual field. Tremor can 
be palpable on patent ductus arteriosus. Pull the lung anteriorly to expose the mediastinum and use wet gauze to protect the lung tissue. The mediastinal pleura is incised along the midline of the longitudinal axis of the descending aorta, from above the origin of the left subclavian artery to the level of the left hilum. (Figure 1) Free the inferior fossa, upper window, and posterior wall of the ductus arteriosus, and guide two 10 gauge silk sutures with a right-angle forcep. (Figure 2) After blocking the ductus arteriosus, the blood pressure rises, and the pulse differential pressure decreases. After lowering the blood pressure, the silk sutures are ligated separately. A slip suture is used to suture and ligate between the two silk sutures. After the tremor disappears, the mediastinal pleura is closed with no active bleeding in the operation field. A chest drainage tube is indwelled, and the chest incision closed layer by layer after the lung is expanded. Sufentanil was used to relieve pain after surgery until the drainage tube was pulled out.

The follow-up schedule of the two groups was similar. After discharge, the clinical data and color Doppler echocardiograms were evaluated at 1 and 6 months and yearly thereafter. All patient families in both groups completed a questionnaire of two simple questions, including "Are you satisfied with the aesthetics of the incision?" and "What bothers you about the incision, the length, the position, or other aspects?"

Variables: The data collected include operation time, intraoperative blood loss, postoperative ventilator assisted ventilation time, postoperative drainage, postoperative hospital stay, postoperative complications, and satisfaction with the aesthetic effect of the incision.

Statistical analysis: In this study, SPSS 19.0 software was used for statistical analysis. A Kolmogorov-Smirnov test was used to test the normality of the measurement data. An unpaired t-test was used to compare continuous variables with a normal distribution between groups, and if the continuous variables or the differences did not conform to the normal distribution, a Wilcoxon signed-rank test was used. The associations between qualitative variables were analyzed by chi-square, continuity correction, or Fisher's exact test. All tests were two-sided, and a probability level less than .05 was used as a criterion of significance.

Baseline data: Patient baseline information is shown in Table 1. (Table 1) Compared with the left posterolateral group, patients in the left axillary group were younger, lower weight, and had more funnel patent ductus arteriosus. There was no significant difference in the proportion of men and the diameter of arterial ductus arteriosus between the two groups.

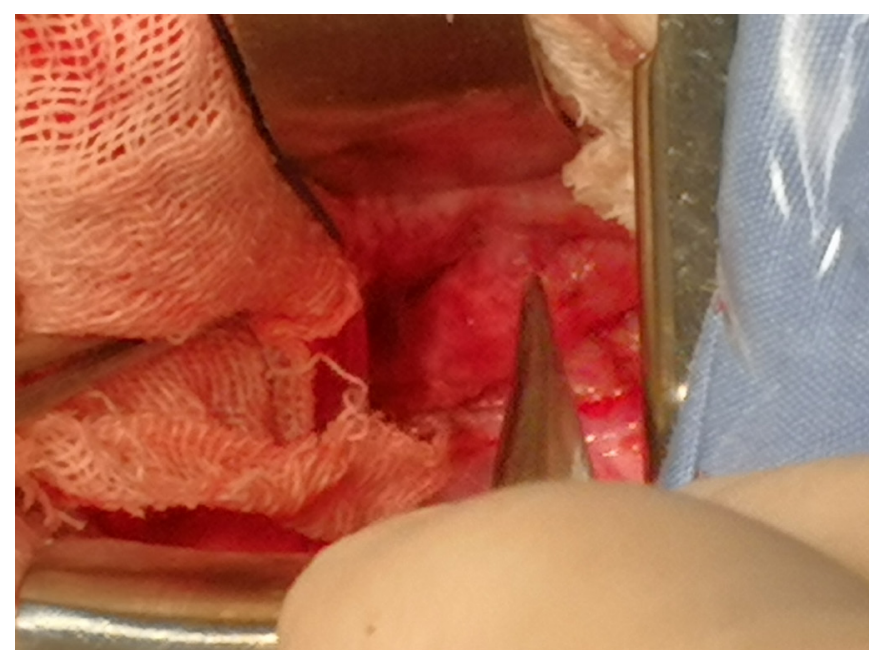

Figure 1. Operative field exposure during left axillary thoracotomy.

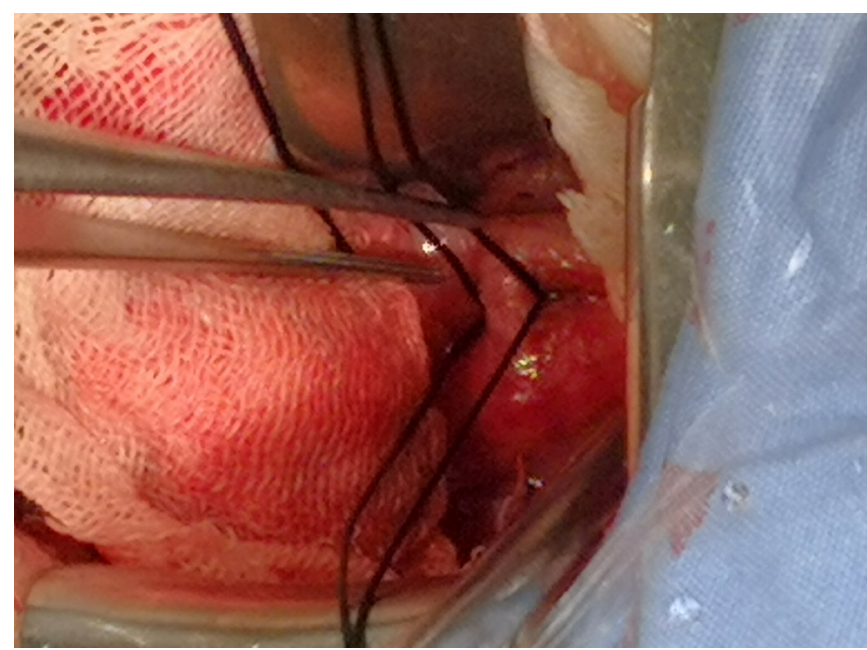

Figure 2. Patent ductus arteriosus exposure before ligated.

Table 1. Comparison of baseline data

\begin{tabular}{lccc}
\hline Items & Left axillary group $(N=76)$ & Left posterolateral group $(N=101)$ & $P$-value \\
\hline Age (years) & $0.58(0.33,0.83)$ & $0.75(0.50,1.71)$ & 0.002 \\
Sex (men/women) & $22 / 54$ & $35 / 66$ & 0.421 \\
Weight (kg) & $6.5(5.5,7.5)$ & $7.4(6.0,10.0)$ & 0.003 \\
PDA size (mm) & $7.0(6.0,10.0)$ & $8.0(6.0,10.0)$ & 0.185 \\
PDA type (tubular/funnel) & $21 / 55$ & $57 / 44$ & 0.000 \\
\hline
\end{tabular}

PDA: patent ductus arteriosus 
Perioperative assessment: The intraoperative and postoperative results of the two groups are shown in Table 2 . (Table 2) There was no need to transfer other incisions in both groups. One case in the left posterolateral group required cardiopulmonary bypass, and the others were completed without cardiopulmonary bypass. There were no significant differences in operation time and intraoperative blood loss between the two groups. The left axillary group had less postoperative drainage, and there was no significant difference in the postoperative ventilator time and postoperative hospital stay between the groups.

Postoperative complications were rare in both groups. In the left axillary group, there were two cases of residual shunt, one case of chylothorax, and one case of atelectasis. In the left posterolateral group, there were three cases of residual shunt, one case of chylothorax, and three cases of atelectasis. There were no complications, such as abnormal incision healing, pneumothorax, re-open for bleeding, and laryngeal nerve injury.

Follow up: The average follow-up time was $22.6 \pm 6.8$ months (range 3-40 months). There were no deaths in either group. In the left axillary incision group, one patient with residual shunt healed spontaneously, and one patient underwent resurgical treatment. All three patients with residual shunt in the left posterolateral group were re-admitted to the hospital for interventional patent ductus arteriosus device closure. In the left posterolateral group, left shoulder lifting range was limited in three patients within three months after surgery. There was no breast asymmetry, thoracic deformity, or scoliosis in either group. The postoperative questionnaire survey showed that $95 \%$ of patients or their families in the left axillary group were satisfied with the aesthetics of the incision, while the satisfaction rate in the left posterolateral group was $80 \%(P=0.007)$.

\section{DISCUSSION}

Patent ductus arteriosus is one of the most common congenital heart diseases. Since Gross successfully completed the first case of arterial ductus ligation in 1938, various surgical methods have been used to close the arterial ductus [Garcia 2017; Susheel 2019]. With the continuous improvement of the safety of cardiovascular surgery, cosmetic results and reduction of trauma have received increasing attention. Surgeons have tried to use various minimally invasive surgical methods to replace conventional surgical incisions and strive to achieve this without affecting the surgical results.

Interventional treatment of patent ductus arteriosus has the advantages of simple operation, high success rate, and fewer complications. In recent years, the interventional closure of patent ductus arteriosus has tended to be the first choice, but its operation needs to be carried out with the aid of imaging, requiring relatively high basic knowledge and proficiency of the operator, and the damage to the child and the operator under X-ray also needs attention [Baruteau 2014; Jin 2015]. Although interventional patent ductus arteriosus closure has less trauma and quicker recovery, it has narrow indications and is expensive [Kim 2019]. It is still contraindicated for young and low birth weight infants [Thanopoulos 2001]. Surgery assisted by thoracoscopy can minimize the surgical incision and has the advantages of beautiful incision, less trauma, light postoperative pain, and quick postoperative recovery [Villa 2004; Gillor 2008]. Thoracoscopic surgery maintains the integrity of the thoracic cage and avoids the adverse consequences of thoracic asymmetry, pterygoid scapula, scoliosis, and asymmetry of breast development that may be caused by extensive damage to the chest wall muscles [Nezafati 2011]. The limitation lies in the lack of flexibility in the operation under thoracoscopy. The operation instruments can only

Table 2. Comparison of perioperative data

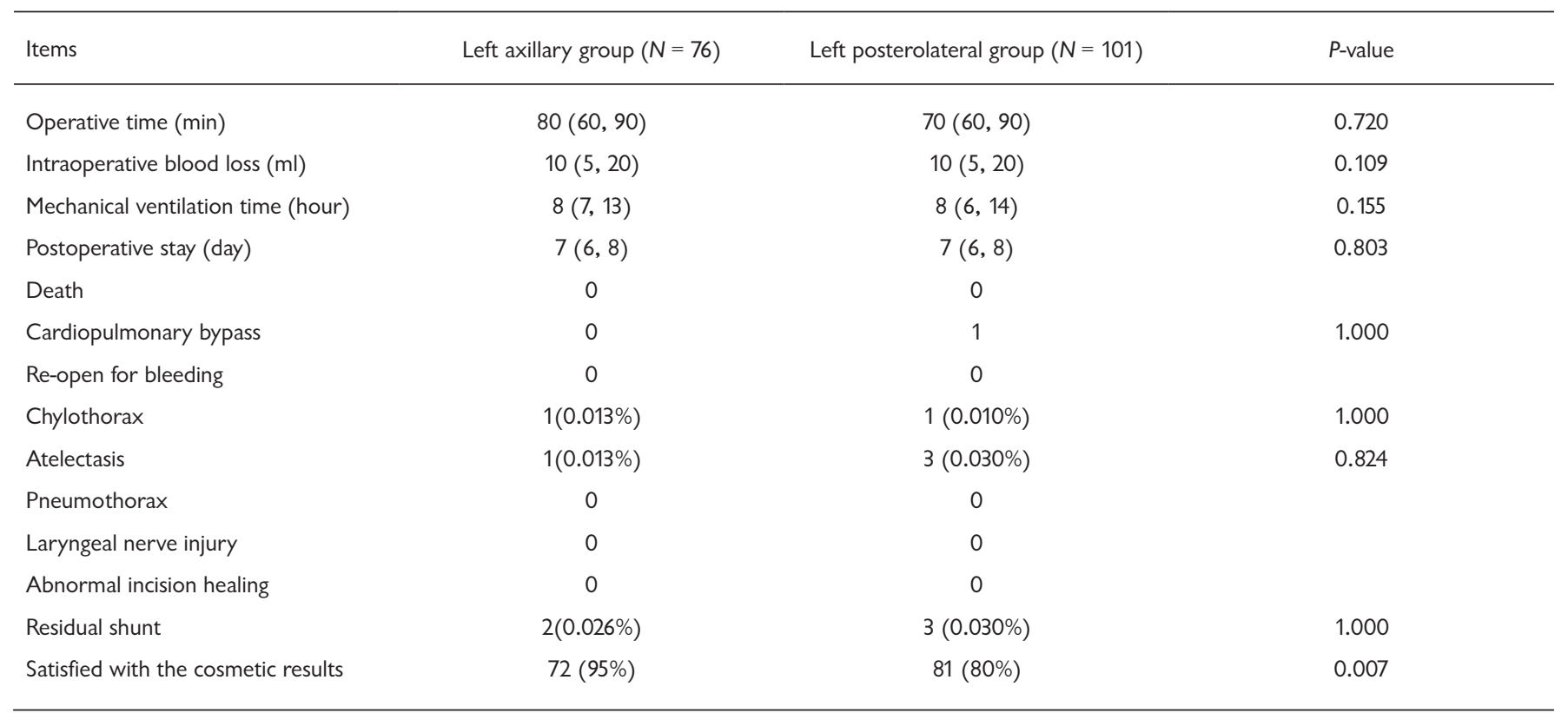


be clamped through the tip. Suture and knotting are timeconsuming. It requires the surgeon to have a solid foundation in cardiac surgery in order to shorten the operation time and deal with emergencies in time. Circumstances, while being restricted by equipment, etc., the use is restricted in children with small weight [Odegard 2000]. The robotic surgical system has beautiful incisions and can provide highdefinition, three-dimensional, and enlarged surgical fields. Its mechanical arm has a high degree of freedom, and large range of motion in the chest cavity can also eliminate hand shaking and is accurate and flexible. Robots can realize telemedicine and have broad application prospects [Suematsu 2005; Le Bret 2002]. The main limitations at present are the high cost of the technology, no tactile feedback system, and long learning curve, making it difficult to widely develop [Bacha 2014]. The lack of equipment suitable for children's surgery and the small chest volume of children limit the operating space.

Currently, the left posterolateral incision is widely used in patent ductus arteriosus ligation, which has the advantages of good exposure and convenient operation [Heuchan 2014; Susheel 2019]. Once bleeding occurs, it is easy to rescue and stop the bleeding. Its mortality, recanalization rate, and residual rate are very low [Lehenbauer 2018; Reese 2018]. However, the left posterolateral incision is unsightly and takes longer operating time to cut the muscles and get into the chest cavity compared with axillary incision. Severe postoperative pain caused by muscle cutting is not conducive to the exercise of the patient's respiratory function.

Our center has achieved good results with the right axillary incision to treat simple intracardiac malformations [Liu 2000; Yang 2019; Li 2015]. On this basis, the left underarm incision has been used to treat patent ductus arteriosus since 2013. In the early stage, it is only used for preschool children. With the accumulation of experience, the application range of this incision is gradually expanded. Small body weight and adults are not contraindicated in surgery. All ages, cast, funnel, moderate or mild pulmonary hypertension, wide chest in adults, and deep side thoracotomy also can be done. The pulmonary artery suture or patch repair under the median incision and cardiopulmonary bypass is mainly used for patent ductus arteriosus with severe pulmonary hypertension, large window patent ductus arteriosus or patent ductus arteriosus with other intracardiac malformations, and recanalization of PDA. Premature infants, infants and young children, including those with respiratory failure and heart failure, are not contraindicated. Early surgery should be recommended to avoid unnecessary damage.

The left axillary incision has the following advantages. First, the left axillary incision has a good aesthetic effect. This incision is small, located under the armpit, hidden in the position by the resting arm. The incision does not exceed the anterior axillary line, the location is far away from the breast, it does not affect breast development, and avoids postoperative breast asymmetry in female patients. Second, the chest wall under the armpit is thin and there are no large muscle groups. Through the incision here, it can quickly enter the chest cavity, which can shorten the operation time and reduce the trauma. The conventional left posterolateral incision also cuts the latissimus dorsi and a small amount of trapezius muscle in addition to the intercostal muscles. The incision is longer and may cause thoracic deformities. In contrast, the left axillary incision into the chest damages less muscles, and there will be no problems, such as left shoulder sinking and passive posture protruding behind the subscapular angle after the left posterolateral incision. The surgical trauma is small, postoperative recovery is quick, postoperative pain is less, intraoperative bleeding and postoperative drainage are less, and blood transfusion is not required. Enter the chest here away from the front and rear rib joints, and the range of motion is large, and joint dislocation is not easy [Prêtre 2005]. Third, the left axillary incision surgery does not require special equipment and materials. The operation is performed under direct vision, no additional cost or training is required, and it has a wide range of applications.

During the operation, attention should be paid to the following aspects: the left axillary incision is usually used for children with the third intercostal space to enter the chest, and the fourth intercostal space is easily exposed for patients older than 10 years. Adults or older children use dual-lumen endotracheal intubation and single-lung ventilation to help expose. It should be noted during the operation that the mediastinal pleura is cut longitudinally, from the left subclavian artery to the left lung hilum. Try to expose and protect the left recurrent laryngeal nerve. Do not pull the vagus nerve and the recurrent laryngeal nerve excessively at this time, otherwise it may cause injury and cause hoarseness after surgery. Strip the loose tissue along the side of the aorta and suspend it on the inside. It can expose the ductus and protect the recurrent laryngeal nerve and expose the space between the lower window of the patent ductus arteriosus. When threading and ligating the knot on the side of the pulmonary artery, only the mediastinal pleura should be lifted. Avoid the recurrent laryngeal nerve under sight. After separating the upper and lower gaps, the small right-angle cyanosis passes from top to bottom. If the ductus is too large and it is expected to be difficult to separate, the proximal and distal thoracic aorta can be cuffed separately in order to block the aorta in case of bleeding from the PDA to control fatal bleeding. Before ligation, deepen anesthesia or use antihypertensive drugs, such as sodium nitroprusside, to reduce blood pressure to reduce the risk of ductus rupture and bleeding. When ligating, use silk thread to ligate the arterial ductus with moderate force. When pulling out the silk thread, the action should be gentle, and the angle of the thread should be expanded to prevent shearing force from tearing the ductus wall. Excessive force or improper force may cause severe bleeding from the cutting or tearing of the arterial ductus. If it is loose, there may be residual shunt from the duct. First, ligate the aortic side first, apply slowly and firmly, tie the knot in place, and do not pull at an angle. If combined with pulmonary hypertension, the first knot can be tied only $80 \%$ to $90 \%$, as appropriate, and the second is close to the pulmonary artery, and the ligation line must be absolutely tight. The distance between the two ligation silk threads should be increased as much as possible to enhance the atresia of the patent ductus arteriosus. After the ligation, a slip thread is used to penetrate the suture 
between the two threads to reduce residual shunt and postoperative ductus recanalization. Early postoperative atelectasis occurs in children. After taking out the gauze before closing the chest, pay attention to fully expand the lungs until all lung tissues are fully expanded.

Our study had some limitations. First, this study was an observational retrospective study and a single-institution survey. Second, no data concerning levels of pain were obtained. Third, compared with the left posterolateral incision, the left axillary incision for patent ductus arteriosus ligation has limited exposure to the surgical field, so this technique is most suitable for preschool children. Surgeons should practice this technique repeatedly in preschool children before expanding the application range to infants and adults in order to avoid additional risks.

\section{CONCLUSION}

Patent ductus arteriosus ligation through the left axillary incision is safe, feasible, and aesthetically effective. The left axillary incision can be used as an alternative to the left posterolateral incision to treat patent ductus arteriosus, which has good clinical promotion value.

\section{REFERENCES}

Bacha E, Kalfa D. 2014. Minimally invasive paediatric cardiac surgery. Nature reviews Cardiology. 11(1):24-34.

Baruteau AE, Hascoet S, Baruteau J, Boudjemline Y, Lambert V, Angel CY, et al. 2014. Transcatheter closure of patent ductus arteriosus: past, present and future. Archives of cardiovascular diseases. 107(2):122-32.

Dice JE, Bhatia J. Patent ductus arteriosus: an overview. 2007. J Pediatr Pharmacol Ther. 12(3):138-146.

Garcia AV, Lukish J. 2017. Minimally Invasive Patent Ductus Arteriosus Ligation. Clin Perinatol. 44(4):763-771.

Gillor A. 2008. Evolution of strategies for management of the patent arterial duct. Cardiology in the young. 18(3):358; author reply 9-60.

Heuchan AM, Clyman RI. 2014. Managing the patent ductus arteriosus: current treatment options. Arch Dis Child Fetal Neonatal Ed. 99(5):F431-F436.

Jin M, Liang YM, Wang XF, Guo BJ, Zheng K, Gu Y, et al. 2015. A Retrospective Study of 1,526 Cases of Transcatheter Occlusion of Patent Ductus Arteriosus. Chinese medical journal. 128(17):2284-9.

Kim HS, Schechter MA, Manning PB, et al. 2019. Surgical Versus Percutaneous Closure of PDA in Preterm Infants: Procedural Charges and Outcomes. J Surg Res. 243:41-46.

Le Bret E, Papadatos S, Folliguet T, Carbognani D, Petrie J, Aggoun Y, et al. 2002. Interruption of patent ductus arteriosus in children: robotically assisted versus video thoracoscopic surgery. The Journal of thoracic and cardiovascular surgery. 123(5):973-6.

Lehenbauer DG, Fraser CD 3rd, Crawford TC, et al. 2018. Surgical Closure of Patent Ductus Arteriosus in Premature Neonates Weighing Less Than 1,000 grams: Contemporary Outcomes. World J Pediatr Congenit Heart Surg. 9(4):419-423.

Li G, Su J, Fan X, et al. 2015. Safety and Efficacy of Ventricular Septal Defect Repair Using a Cosmetic Shorter Right Lateral Thoracotomy on Infants Weighing Less than $5 \mathrm{~kg}$. Heart Lung Circ. 24(9):898-904.

Liu YL, Zhang HJ, Sun HS, et al. 2000. Repair of cardiac defects through a shorter right lateral thoracotomy in children. Ann Thorac Surg. 70(3):738-741.

Mitra S, Rønnestad A, Holmstrøm H. 2013. Management of patent ductus arteriosus in preterm infants--where do we stand? Congenit Heart Dis. 8(6):500-512.

Nezafati MH, Soltani G, Kahrom M. 2011. Video-assisted thoracoscopic patent ductus arteriosus closure without tube thoracostomy. The Annals of thoracic surgery. 91(5):1651.

Odegard KC, Kirse DJ, del Nido PJ, Laussen PC, Casta A, Booke J, et al. 2000. Intraoperative recurrent laryngeal nerve monitoring during video-assisted thoracoscopic surgery for patent ductus arteriosus. Journal of cardiothoracic and vascular anesthesia. 14(5):562-4.

Prêtre R, Kadner A, Dave H, Dodge-Khatami A, Bettex D, Berger F. 2005. Right axillary incision: a cosmetically superior approach to repair a wide range of congenital cardiac defects. J Thorac Cardiovasc Surg. 130(2):277-281

Reese J, Scott TA, Patrick SW. 2018. Changing patterns of patent ductus arteriosus surgical ligation in the United States. Semin Perinatol. 42(4):253-261.

Schneider DJ. 2012. The patent ductus arteriosus in term infants, children, and adults. Semin Perinatol. 36(2):146-153.

Suematsu Y, Mora BN, Mihaljevic T, del Nido PJ. 2005. Totally endoscopic robotic-assisted repair of patent ductus arteriosus and vascular ring in children. The Annals of thoracic surgery. 80(6):2309-13.

Susheel Kumar TK. 2019. Surgical management of patent ductus arteriosus. Congenit Heart Dis. 14(1):57-59.

Thanopoulos BD, Hakim FA, Hiari A, Tsaousis GS, Paphitis C, Hijazi ZM. 2001. Patent ductus arteriosus equipment and technique. Amplatzer duct occluder: intermediate-term follow-up and technical considerations. Journal of interventional cardiology. 14(2):247-54.

Villa E, Vanden Eynden F, Le Bret E, Folliguet T, Laborde F. 2004. Paediatric video-assisted thoracoscopic clipping of patent ductus arteriosus: experience in more than 700 cases. European journal of cardio-thoracic surgery: official journal of the European Association for Cardio-thoracic Surgery. 25(3):387-93.

Yang M, Su J, Liu A, et al. 2019. Correction of simple congenital heart defects by right axillary thoracotomy in adults. J Card Surg. 34(11):1172-1177. 